\title{
Las habilidades sociocognitivas como herramientas en el proceso de mediación ${ }^{*}$
}

\author{
María José Briz Clariget \\ Universidad Católica del Uruguay, Montevideo, Uruguay \\ mbriz@ucu.edu.uy \\ https://orcid.org/0000-0002-7620-8073
}

\section{RESUMEN}

En este trabajo se realiza un análisis de materia y un análisis crítico de la informalas habilidades sociocognitivas como herramientas que deben ser utilizadas por el mediador en el proceso de mediación y ejercitadas con los participantes para poder gestionar los conflictos en forma constructiva. La metodología utilizada se centrará en un análisis doctrinario de la ción obtenida a los efectos de generar un aporte para que el tema sea enriquecido por la formación y experiencia práctica de la autora en el área.

Palabras clave: solución de conflictos; arbitraje; resiliencia; escucha activa; competencias para la vida.

Cómo citar: Briz, M. (2021). Las habilidades sociocognitivas como herramientas en el proceso de mediación. Ciencias Sociales y Educación, 10(19), 167-188. https://doi.org/10.22395/csye.v10n19a7

Recibido: 30 de noviembre de 2020.

Aprobado: 3 de marzo de 2021. 


\section{Social and Cognitive Skills as Tools in the Mediation Process}

\section{ABSTRACT}

This work analyzes the social and cognitive skills as tools for the use of mediators in the mediation process and employed by its participants to solve conflicts in a constructive way. The methodology employed in the research was centered on a doctrine analysis on the matter and a critical analysis of the obtained information with the objective of generating a contribution to the topic and its enrichment with new information and practical experience of the author in this area.

Keywords: conflict resolution; arbitration; resilience; active listening; life skills.

\section{As habilidades sócio-cognitivas como instrumentos no processo de mediação}

\section{RESUMO}

Neste trabalho realiza-se uma análise das habilidades sócio-cognitivas como instrumentos que devem ser utilizados pelo mediador no processo de mediação e exercê-las com os participantes para poder gerenciar os conflitos de maneira construtiva. A metodologia utilizada centrar-se-á na análise doutrinária da matéria e uma análise crítica da informação obtida aos efeitos de gerar uma contribuição para que o tema seja enriquecido pela formação e experiência prática da autora na área.

Palavras chaves: solução de conflitos, arbitragem, resiliência, escuta ativa, competências para a vida. 


\section{Introducción}

En el ámbito de las interacciones humanas, el conflicto se presenta en forma constante en distintos niveles y con diferentes características. En la inmensa mayoría de los casos, la comunicación juega un papel muy importante en su gestación y es el origen del conflicto. Por lo tanto, si pensamos en una gestión constructiva de los conflictos que se presentan, es fundamental trabajar en la comunicación interpersonal. Las personas se comunican como pueden y como saben hacerlo. Las técnicas de comunicación se adquieren desde el momento en que la persona nace, y evolucionan de acuerdo con su contexto familiar, educativo y social.

En el campo de la mediación como método alternativo de resolución de conflictos es fundamental el nivel de desarrollo de las habilidades con las que las partes se enfrentan al problema que se les plantea y la capacidad que tienen de generarlas dentro el mismo proceso. Por otro lado, es fundamental el desarrollo que el mediador tenga de estas capacidades y el nivel de formación que posea para hacer que los participantes, durante el proceso — si no tuvieran alguna habilidad que fuere necesaria para gestionar constructivamente el conflicto al que se enfrentan-, puedan desarrollarla a través de la aplicación, por parte del mediador, de las técnicas específicas del ámbito de la mediación.

Es importante destacar que estas habilidades deben trabajarse desde el momento del nacimiento de cualquier persona. Los diferentes programas en el ámbito educativo y social deberían acompañar su desenvolvimiento y potenciar el desarrollo que se pueda lograr en el ámbito educativo y familiar. Si bien son capacidades que a la hora de gestionar constructivamente conflictos se vuelven imprescindibles, también son necesarias para que las personas puedan construir vínculos positivos con los demás y desarrollar su vida en un ambiente saludable. Esto no quiere decir que contar con ellas asegure no tener conflictos, ya que el conflicto es inherente al ser humano, sino que implica el modo en que la persona se posiciona frente a los conflictos, el grado de adaptación tiene a los cambios y la calidad de interacción ostenta a nivel social.

El análisis de estas habilidades, enmarcadas en el proceso de mediación, genera una verdadera gestión constructiva y asegura un proceso transformativo de las partes, pues aunque no es estrictamente necesario lograr un acuerdo para generar transformación, si este se alcanza, se logrará un alto grado de cumplimiento.

Desde la perspectiva del mediador, tradicionalmente se ha destacado la importancia de que esté capacitado en el uso de las herramientas específicas en el ámbito de la mediación, pero desde hace poco tiempo se comenzó a visualizar la relevancia que tiene contar con un manejo adecuado de las habilidades sociocognitivas desde su rol y como encargado de que las partes las desarrollen dentro 
del proceso de mediación. Es habitual que se vincule la importancia de dichas habilidades a ámbitos de gestión de conflictos en los que el componente emocional es más alto, como en el terreno familiar o el comunitario. Sin embargo, actualmente se considera que su desarrollo es vital para gestionar cualquier tipo de conflicto dentro de un proceso de mediación con el fin de generar una transformación en las personas que forman parte de él.

\section{Las habilidades sociocognitivas}

Partiendo de la base de que para poder vivir en una sociedad pacífica es fundamental el comportamiento de cada una de las personas que forman parte de ella, es indispensable visualizar cuáles son las características que debe tener un individuo para poder lograr comportamientos constructivos en la gestión de sus conflictos.

Según lo que sostienen Pesqueira y Ortiz (2018):

Las habilidades socio-cognitivas son indispensables para aprender a vivir juntos, respetando la dignidad intrínseca de la que irradian los derechos fundamentales de cada ser humano, siendo en este contexto que para gestionar exitosamente los conflictos es necesario el desarrollo de dichas habilidades. Ya que su puesta en práctica, cuando nuestros intereses colisionan con los de otros, nos permite trabajar sinérgicamente en la búsqueda de soluciones que produzcan en los protagonistas la percepción objetiva de que el conflicto ha sido resuelto. (p. 257)

Si bien las habilidades sociocognitivas tienen una base biológica en el cerebro, permanecen en estado latente si, en el proceso de desarrollo como ser humano, no son tenidas en cuenta.

Según Myers, citado por Abello, Amar y Tirado (2014), el desarrollo humano:

Es multidimensional, porque incluye el mejoramiento de un conjunto interrelacionado de dimensiones. Es potencialidad, lo que implica siempre llegar a ser más. Es un proceso continuo, que comienza antes del nacimiento y se prolonga a lo largo de toda la vida. Es un proceso integral, pues los diferentes elementos del desarrollo humano están interrelacionados y deben ser considerados como un todo. Es adaptativo, porque implica preservar el sentido histórico del individuo y al tiempo potencializar su capacidad de cambio. (p. 4)

Por este motivo, resulta fundamental tener en cuenta el desarrollo de estas habilidades en el proceso de crecimiento del ser humano, así como las físicas, lingüísticas, estéticas o ético-morales, siempre considerando que su capacidad de progreso es inagotable.

Las habilidades sociocognitivas son capacidades con las que el individuo nace. Al desarrollarlas, hacen que este se vuelva competente y le permiten esta- 
blecer interacciones sociales constructivas con las personas que lo rodean y con la sociedad en general. Su desenvolvimiento depende de los procesos de aprendizaje que se dan en el inicio de su desarrollo dentro del ámbito familiar. Luego pasa gradualmente a depender de otros ámbitos, como el educativo, el social, el laboral, entre otros en los que el individuo se encuentra inmerso. Son habilidades que generan capacidades muy básicas, como permitirle discriminar entre objetos, estímulos y que, a través del crecimiento, se vuelven capacidades más complejas que permiten resolver de manera positiva los diferentes conflictos generados en la interacción permanente del individuo en el transcurso de su vida.

Según Gatti (2005, p. 124), uno de los abordajes clásicos más completos de las habilidades sociocognitivas lo desarrolló Paul Guilford en el año 1967. Este las organizó en un modelo de rejilla de cubo tridimensional en el que se encuentran comprendidos tres elementos: operaciones (cognición, memoria, pensamiento divergente, pensamiento convergente y evaluación), productos (unidades, clases, relaciones, sistemas, transformaciones, implicaciones) y contenidos (figurativo, simbólico, semántico y comportamental). Este cubo genera ciento veinte celdas que pueden ser combinadas entre ellas de forma fluida, flexible y original. La importancia de este modelo, en el marco de este análisis, es la creación de lo que denominó "pensamiento divergente", el cual se sitúa en el ámbito del pensamiento creativo. Esta habilidad es fundamental a la hora de enfrentar un conflicto.

El pensamiento divergente se caracteriza por la flexibilidad espontánea, que es lo que le permite a quienes gestionan conflictos, por ejemplo, pedirles a las partes generar posibles alternativas para la situación planteada.

Gatti (2005) también afirma que muchos especialistas que han desarrollado el tema consideran que el procesamiento de la información es parte de la base de las habilidades cognitivas. Esta es una habilidad básica para la vida de cualquier individuo en la sociedad en la que estamos inmersos, en la que, de alguna manera, estamos intoxicados por la cantidad de información. Por ello, cada vez es más difícil su procesamiento y más profundo el impacto que esta tiene sobre la toma de decisiones (Gatti, 2005, p. 127).

\section{Las habilidades sociocognitivas y la gestión de conflictos}

Es evidente que el grado de desarrollo de las habilidades sociocognitivas que tenga el individuo condicionan la manera en que este se posiciona frente a una situación conflictiva. Todas las personas ostentan actitudes y estrategias que las predisponen, ante situaciones generadoras de desequilibrio, a lograr prevenir el conflicto o gestionarlo de manera constructiva. La gran destreza que debe tener el mediador, desde su perspectiva de mirada amplia, es saber utilizar la 
habilidad y la técnica precisa en el momento justo del proceso para que esta impacte positivamente. Melendro y Monserrat (2017), en una investigación realizada para detectar las habilidades más relevantes que se deben desarrollar en los adolescentes para mejorar su inclusión social en contextos vulnerables, destacan el rol fundamental de las habilidades sociocognitivas.

Según Camacho y Céspedes (2018), las habilidades sociocognitivas son necesarias para enfrentar con éxito los desafíos que la vida presenta a diario. Las autoras mencionan algunas "habilidades para la vida" (propuestas por la Organización Mundial de la Salud, OMS) — como la empatía, la comunicación asertiva, el pensamiento creativo, el razonamiento crítico, entre otras - con las que es relevante que las personas puedan contar en cualquier contexto sociocultural. La investigación constata la necesidad de que los mediadores policiales cuenten con habilidades sociocognitivas y afirma que su desarrollo aporta al propósito ulterior de la construcción de paz y convivencia familiar pacífica.

Echeverria et al. (2020) destacan la importancia de estas habilidades a la hora de conseguir empleo, lo que condiciona su incidencia en el grado de desarrollo laboral y oportunidades laborales de una persona.

Ortiz y Gaeta (2019) refieren que en un programa de educación elaborado desde la Universidad de Barcelona se propone el desarrollo socioemocional de los estudiantes mediante el trabajo en el aula a partir de ciertas habilidades, entre las que se encuentran las "habilidades para la vida y el bienestar, destinadas a una utilización focalizada en la solución de problemas" (p. 151).

Por otro lado, Caballo (2007), en su investigación sobre la evaluación y desarrollo de las habilidades sociales, incluye las habilidades para solucionar problemas que

corresponden a la capacidad del niño para solucionar de una manera adecuada, y por sí mismo, los problemas interpersonales que se le presentan con otros niños [...] entendiendo que la relación del niño con el adulto es distinta a la que se da con otros niños y abarca también las habilidades del niño para relacionarse con el adulto mediante conversación, cortesía, peticiones, refuerzo y solución de problemas. (p. XIV)

Analizaremos en detalle algunas de estas habilidades y sus características principales.

\section{La empatía}

Según Rogers (año), citado en Gelsi (2011, p. 121), la "comprensión empática" es la aptitud que tiene una persona - en este caso el mediador como gestor de conflictos- de percibir y sentir el mundo interno del otro como si fuera el propio. Este fenómeno se presenta en el proceso de gestión de conflictos tanto 
en quien tiene la tarea de orientar el proceso (el mediador) como en las partes que intervienen.

El uso de la fórmula como si dotará de profesionalismo la intervención, ya que quien está ejerciendo la tarea, como en el caso del mediador, debe analizar el conflicto con los ojos de la otra persona para conocer cómo lo siente y cómo lo vive. De esta manera, se implica en ello, pero de manera profesional, es decir, sin reconocerlo como propio, sin perder nunca la conciencia de su individualidad. La conservación de la distancia emocional entre el mediador y las partes es vital para que aquel pueda cumplir con su rol de forma competente. Tal como afirma Rogers (año), el profesional que reconoce con claridad el mundo del cliente está en disposición de hacer llegar su comprensión (citado en Gelsi, 2011, p. 121).

Camacho y Céspedes (2018) también manifiestan la importancia de la empatía como habilidad fundamental en el mediador policial, ya que contribuye a generar una buena comunicación en el marco de la solución de problemas y conflictos.

El momento en que el mediador puede llegar a transmitir a las partes esta habilidad sociocognitiva (la empatía) es cuando aplica la técnica del parafraseo o a través de las preguntas para tratar de trasladar lo que comprendió de la situación planteada.

\section{La escucha activa}

Otra habilidad indispensable para el mediador, y que es muy importante desarrollar entre las partes o con el propio mediador, es la escucha activa. Esta habilidad implica escuchar al otro, pero no para responder, sino para comprender lo que está diciendo. Esta habilidad es vital para ejercer el rol del mediador. A través de esta, este actúa como un amplificador de los pensamientos de las partes y logra que ellas se los replanteen para reconsiderar sus propias posiciones. La escucha activa es una habilidad comunicacional que se apoya en muchas otras técnicas derivadas de las ciencias de la comunicación para, de alguna manera, poder comprender y dar respuesta a las necesidades de las partes en un proceso de mediación. Mullender (año) manifiesta que "casi todos pensamos que sabemos escuchar, pero no es así. La mayor parte de la gente no lo hace con la intención de comprender, sino de responder" (citado en lese Insight, 2014, p. 70).

La pregunta es una de las técnicas más utilizadas en todo proceso de mediación y la clasificación más general que puede realizarse, teniendo en cuenta su construcción y su finalidad, es entre preguntas abiertas y cerradas. La pregunta abierta es aquella que busca obtener información extensa sobre determinados hechos, pero se debe tener mucho cuidado al utilizarla, ya que deja 
libres a las partes de hacer comentarios defensivos o acusatorios que hay que tratar de evitar en un proceso de mediación. Las preguntas cerradas, en cambio, van más direccionadas a obtener determinado dato preciso sobre un hecho concreto. Son útiles en procesos en los que el detalle es fundamental para gestionar el conflicto, pero son peligrosas porque muchas veces se puede traslucir a través de ellas la hipótesis del mediador sobre los hechos, lo que pondría en tela de juicio su neutralidad (Caram, 2006, p. 235).

Las oportunidades en las que se utiliza uno u otro tipo de preguntas son muy variadas, pero las preguntas cerradas son muy aplicadas en la etapa de cerrar acuerdos, mientras que las preguntas abiertas son sumamente oportunas en aquellos momentos en los que los mediadores están tentados a perder la neutralidad y emitir un juicio de valor o, sobre todo, en los casos en los que la profesión de origen permite brindar asesoramiento.

Otro tipo de preguntas muy útiles son las circulares, que llevan a las partes a reflexionar sobre el asunto, y tienen como objetivo que una se ponga en el lugar de la otra. Muchas veces son la vía para usar la técnica denominada abogado del diablo. Las preguntas, en general, logran encontrar información que no estuvo presente en la narrativa de las partes. Esta queda — de alguna manera- perdida en el proceso y es relevante para la gestión del conflicto. Por otro lado, identifican las distorsiones, eliminaciones y generalizaciones, así como permiten descubrir y dejar en evidencia el mapa del conflicto y de cada una de las partes que intervienen en el proceso. Adicionalmente, la pregunta genera un cambio en la atención de las partes y en cómo se sienten en el proceso. En este sentido, permite recuperar aquello que muchas veces se ha suprimido de la conciencia y cambia los recursos de los que se disponen a seguir gestionando el conflicto (Balletto, Briz y Falca, 2019, p. 143). Sin embargo, según Mullender (año, citado en Iese Insight, 2014), no es conveniente realizar muchas preguntas cuando se está escuchando activamente, ya que cada vez que interrumpimos corremos el riesgo de desviar el tema.

A través de la pregunta se logra la escucha activa y el mediador puede comprender lo que se dice, esto es, el contenido del conflicto, lo que provoca cada actor (lo que la pregunta suscita en una parte, en la otra y en el mediador) y cómo se dice o no se dice (la forma de expresarse que tienen las partes, sus gestos, miradas, posturas, tonos de voz, entre otros aspectos). Es fundamental que el mediador logre esta habilidad, ya que le permite conocer lo que le preocupa a cada participante o lo que quiere expresar, para poder entender qué es lo que lo lleva a tener determinada posición. Si el mediador logra manejar esta habilidad de forma eficiente, tanto en las sesiones conjuntas como en las privadas, podrá 
detectar cuál es la posición y el interés de cada parte para comenzar a trabajar sobre los intereses y dejar de lado las posiciones.

Para las partes, lograr la escucha activa del otro es fundamental porque, en la mayoría de las situaciones conflictivas, los participantes no se sienten escuchados ni tampoco son capaces de escuchar activamente a la contraparte. Es por este motivo que, en muchas ocasiones, el gran desafío para el mediador es gestionar un vínculo conflictivo en el marco de una mala comunicación y no tanto el conflicto en sí mismo. Mullender (año, citado en lese Insight, 2014) manifiesta que es una habilidad sociocognitiva difícil de lograr, ya que no solo se debe escuchar, sino interpretar y después poner a prueba esa interpretación, en el caso del mediador, cuando se realiza el parafraseo. Si este es correcto, la persona se va a sentir escuchada, pero si lo que interpreta no es acertado, se ha fallado en la escucha activa y esto puede generar desconfianza a la parte en el proceso.

\section{El autocontrol}

El conflicto siempre está rodeado de emociones, por lo que están presentes, en su gestión, durante todo el proceso y en todas las partes.

Etimológicamente, el término emoción proviene de movere que significa 'moverse'. Al añadirle la e inicial, quiere decir 'moverse hacia' (Goleman, 1995, p. 331).

Para algunos autores, "la emoción es un estado complejo que incluye una percepción acentuada de una situación y objeto, la apreciación de su atracción o repulsión consciente y una conducta de acercamiento o aversión" (FernándezAbascal, Jímenez y Martín, 2003, p. 5). Para Sellers (2019), "las emociones son esos sentimientos y apetitos que nos mueven a la acción dentro de sus propias posibilidades" (p. 11). Entonces, tanto quienes gestionan conflictos como quienes están involucrados en ellos deben saber manejar sus emociones a través del autocontrol. Dicha habilidad implica que el individuo sea capaz de darse cuenta de lo que debe hacer o no para no dañar al otro; pero, para lograrlo, es necesario el autoconocimiento. Esto requiere recorrer el camino de la conciencia propia y amigarse con las cualidades positivas personales que permiten abstenerse de hacer lo que no es bueno para uno ni para quienes lo rodean (Pesqueira y Ortiz, 2018, p. 23).

El autocontrol es vital para la rutina diaria y también lo es cuando se gestionan los conflictos propios. Específicamente dentro de un proceso de mediación, es fundamental que tanto las partes como el mediador puedan desarrollar esta habilidad para lograr un abordaje constructivo del proceso. La importancia de esta habilidad se acentúa más cuando los conflictos que se gestionan revisten 
un alto contenido emocional, como en el caso de los procesos de mediación en el ámbito familiar, en los que la parte está impregnada de emociones a la hora de realizar la narrativa, escuchar al otro, tomar decisiones y cumplir acuerdos.

Camacho y Céspedes (2018) hacen especial hincapié en esta habilidad al analizar al mediador policial,:

se requiere potenciar a través de los procesos de formación y especialización en temas de mediación policial, en relación a la capacidad para expresar sus emociones, seguido del reconocimiento de emociones y sentimientos propios y de los demás y la regulación emocional.

Y por otra parte, con el manejo de tensiones y estrés [...] sin embargo, es de gran importancia que en los procesos de formación y especialización [...] se desarrollen capacidades relacionadas con el reconocimiento de fuentes y efectos del estrés así como la búsqueda de soluciones antes situaciones estresantes. (p. 292)

\section{La compasión}

La compasión, en tanto habilidad sociocognitiva, es considerada como el sentimiento de tristeza que produce ver padecer a alguien y que impulsa a aliviar su dolor o sufrimiento, a remediarlo o a evitarlo. Es una habilidad con la que se nace y que es inherente a la persona, pues es innato ayudar a alguien que está en riesgo o tratar de evitarle el mal a otro. Esto surge de la compasión que el ser humano siente por sí mismo, es decir, todos tratamos de cuidarnos a nosotros mismos y de evitar acontecimientos que nos generen tristeza o dolor. Y desde el sentir compasión por nosotros mismos surge el sentimiento de compasión por los demás (Pesqueira y Ortiz, 2018, p. 268).

Sin embargo, el contexto ha llevado a que, cada vez más, el ser humano pierda esta habilidad que es tan innata de su ser, ya que se estimula el individualismo, el sentimiento de indiferencia sobre el mal de otro y a que se vuelva más selectivo acerca de por quiénes siente compasión. Muchas veces acontece a nivel familiar. La falta de compasión de las generaciones más jóvenes hacia los adultos mayores es un tema de gran actualidad que llega, incluso, al desprecio, lo que genera conflictos familiares que son de alta complejidad a la hora de su gestión.

Es fundamental que en los diferentes procesos educativos y sociales en los que tiene lugar el desarrollo del individuo se mantenga presente trabajar dicha habilidad. Desde la escuela hasta la universidad, en el ámbito familiar, desde que la persona nace, durante el transcurso de toda su vida y también en el ámbito laboral —donde constantemente se revelan entornos cada vez más competitivosse debería trabajar para que las personas logren ser compasivas con el otro para impactar positivamente en la gestión de sus conflictos. 
Walters (2020, pp. 64-67) incluye la compasión entre las cuatro habilidades que debería tener un líder: colaboración, coraje, pragmatismo y compasión por sí mismo y por los demás. Walters estima necesario que el líder esté atento a lo que lo afecta y a lo que afecta al resto de sus compañeros. Hirsch, Izarra y Navia (2020, p. 13) consideran esta habilidad dentro de las que debería tener el docente universitario para poder ejercer su rol con calidad.

Louidor (año), citado en Latorre (2020, p. 93) manifiesta, en referencia a la situación actual de pandemia a nivel mundial, la relevancia de estas habilidades que, sin lugar a duda, se han hecho más visibles en este escenario de crisis. En este sentido, Louidor (año) manifiesta:

Se han caído las pequeñas burbujas y nos hemos encontrado con otro mundo que no era el que esperábamos. Ahora nos damos cuenta de que la mayoría del mundo sigue siendo pobre, carente de bienes básicos, sin oportunidad de tener posibilidades para una vida digna. Es la hora de recuperar la dolencia humana, la compasión que brota de una auténtica fraternidad que no se basa en la simpatía o empatía con unos o algunos, sino que apuesta por la humanización de todos por igual, incluso desgastando la propia vida en ello. (Citado en Latorre, 2020, p. 93)

En la gestión de conflictos y para el perfil del mediador es vital poder contar con esta habilidad, ya que genera un contexto favorable para que tengan lugar otras habilidades, como la empatía, la escucha activa y la asertividad.

Campos et al. (2014), siguiendo a Figley (2014), hacen referencia al fenómeno de fatiga por compasión, con el que se ven afectados muchos mediadores, y hacen hincapié en que "cuanta más exposición al cliente que sufre, mayor es la respuesta empática; cuanto mayor es el estrés por compasión residual, menor es la resiliencia a la fatiga por compasión" (p. 13).

\section{El pensamiento creativo}

Cuando las personas se encuentran frente a un conflicto y buscan ayuda para gestionarlo, por lo general se debe a que no ven la salida o encuentran una única solución que es contraria a la visualizada por la otra parte afectada. Por este motivo, es fundamental que quien gestione dicho conflicto trabaje sobre esta habilidad denominada pensamiento creativo.

El pensamiento creativo consiste en el desarrollo de nuevas ideas y conceptos. Se trata de la habilidad de formar originales combinaciones de ideas para llenar una necesidad. Esta habilidad requiere ser aprendida. Por ello, resulta fundamental que se encuentre presente su posibilidad de desarrollo desde edad muy temprana en el individuo. 
La sociedad actual es compleja y dinámica, por lo que todos los conocimientos adquiridos hoy quedarán desactualizados dentro de muy poco tiempo, así que es importante, como individuos, tener la capacidad de pensar creativamente frente a situaciones desconocidas. Es necesario aprender a pensar frente al conflicto, ya que no se traen las soluciones adquiridas para todos los conflictos que se presentan en la vida. Pero, por lo general, la educación lleva siempre al pensamiento convergente, es decir, el que se caracteriza por el uso de la razón, la lógica y la experiencia, y muchas veces se deja de lado el pensamiento creativo o divergente, que es aquel que impulsa una forma de pensar diferente y creativa, especialmente útil para buscar soluciones a nuevos problemas (Font et al., 2020).

Sternberg y O'Hara (2005) estiman un error la baja importancia que se le da a esta habilidad, pues consideran que las personas que serán exitosas en el futuro son aquellas que tienen esta capacidad desarrollada. Aplicando su teoría a la gestión de conflictos, se puede afirmar que es fundamental para quien ayuda a gestionarlos -en este caso, el mediador - tener claro qué impacto tiene poder desarrollar en las partes esta habilidad a la hora de buscar soluciones al conflicto planteado. De acuerdo con esta teoría, existen tres aspectos de la inteligencia de las personas que son clave para la creatividad: las habilidades sintéticas, las analíticas y las prácticas.

La habilidad sintética es la habilidad para generar ideas nuevas, de alta calidad y adecuadas al conflicto planteado dentro del proceso de mediación. La habilidad analítica, según los autores, es la capacidad necesaria para juzgar el valor de las ideas propias y las de los demás, y decidir cuál de ellas es viable a la hora de solucionar un conflicto. Y la tercera y última habilidad dentro del pensamiento creativo es la práctica, que implica saber vender la idea a la otra parte y lograr llevarla a la acción (Sternberg y O'Hara, 2005, pp. 120-122).

La personalidad del individuo tiene conexión con su capacidad de desarrollar dicha habilidad. En este sentido, Barron (1969) manifiesta que "los individuos creativos están muy bien informados, se interesan por los problemas fundamentales, tienen facilidad de expresión, una personalidad bien afirmada, capacidad de iniciativa, son emprendedores, enérgicos y atrevidos" (pp. 45-48).

También el contexto en el que se gestiona el conflicto puede condicionar esta habilidad. Slabbert (1994) sostiene que "la creatividad se manifiesta mejor en ausencia de presiones externas y de control, en un clima de seguridad y confianza" (pp. 60-69). En el contexto que refiere el autor tiene lugar el proceso de mediación, ya que se trata de un procedimiento flexible, en el que se prioriza la confianza y la voluntad de las partes frente a la coerción.

Cuesta y Lora (2015) manifiestan que 
la mediación creativa -en cuanto posibilidad de transformación, de articulaciones novedosas y de nuevas construcciones de sentido que permitan la reinvención de la realidad existente- emerge en el momento en que los sujetos y grupos evidencian nuevas posibilidades de ser y proponen nuevos mundos posibles. (p. 54)

Por este motivo, el proceso de mediación podría ser campo fértil para que surja dicha habilidad, el mediador cuente con ella y pueda trabajarla con ambas partes frente a la gestión de un determinado conflicto.

\section{La asertividad}

La asertividad es la habilidad que tiene el individuo de transmitir a otro lo que cree y piensa en el momento y de la forma adecuada para no agredir.

Por lo general, el individuo, al encontrarse frente a una situación de conflicto, se inhibe o reacciona de forma agresiva. La asertividad es la habilidad que debe tener la persona para no actuar de ninguna de estas maneras, sino para poder transmitir su posición o interés de manera que no afecte al otro y, lo que es más importante, que sea escuchada. Muchas veces, por la falta de esta habilidad, la actitud que toman las personas frente a los conflictos es la inactividad, porque piensan que el conflicto desaparecerá con el paso del tiempo y no tendrán que enfrentarlo, pero de esta forma solo logran que el conflicto escale y que el vínculo entre las partes se deteriore.

Rancer y Avtgis (2006) desarrollan una teoría sobre la comunicación argumentativa y agresiva. En este sentido, establece la diferencia entre una discusión constructiva, basada en esta habilidad sociocognitiva, y una destructiva, basada en la hostilidad. Consideran que, a pesar de que el conflicto es inherente a cualquier persona, la forma de discutir diferencia la manera en que se gestiona ese conflicto: constructiva o destructiva.

Izquierdo (2010) manifiesta que "la comunicación asertiva es una forma de expresión consciente, congruente, clara, directa y equilibrada, cuya finalidad es comunicar ideas y sentimientos; este tipo de comunicación es necesaria y conveniente a causa de los beneficios que genera" (p. 3).

Por otro lado, Martí (2020) afirma:

La asertividad es el arte de expresar nuestras necesidades y opiniones sin ofender a los demás. En cambio, las personas no asertivas suelen pecar, por exceso o por defecto, de un estilo agresivo o sumiso que les lleva a generar conflictos con los demás, tener una baja autoestima y a no lograr sus objetivos. Las personas asertivas se expresan de forma franca y directa pero sin imponer su criterio, y utilizan un lenguaje corporal relajado, un tono de voz firme y un espacio interpersonal adecuado. (p. 23) 
La asertividad está vinculada con la capacidad para defender derechos propios. Por lo tanto, primero hay que tener esa capacidad para luego poder transmitir de manera asertiva lo que se piensa y defenderlo. Según Pesqueira y Ortiz (2018, p. 267), también implica saber extraer lo mejor de las experiencias pasadas para interaccionar con el presente y cicatrizar las heridas que dejan los conflictos vividos para generar un nuevo contexto que permita convivir en forma armónica.

El desarrollo de esta habilidad en el mediador es básico. Si su comunicación no es asertiva con respecto a las partes, será complejo que logre una comunicación eficiente. El mediador debe tener total libertad para expresarle a las partes y a sus letrados, si estuvieran presentes, lo que él percibe y piensa del proceso de una manera sincera y clara. En la necesaria actitud proactiva que debe tener como persona responsable de guiar el proceso, la forma de comunicarse en todas las etapas y en la aplicación de las diferentes técnicas - como en las preguntas o el parafraseo, su comunicación debe ser asertiva. Una técnica puede ser muy buena, pero si se utiliza en un momento que no es el correcto dentro del proceso, seguramente fracasará y esto implica falta de asertividad por parte del mediador.

Dentro su rol, el mediador también tiene el deber de lograr que las partes se comuniquen de la mejor manera posible y de forma asertiva. Cuando las partes se presentan en el proceso de mediación, por lo general, se encuentran en posiciones enfrentadas y, por lo tanto, la comunicación entre ellas es agresiva o puede darse que alguna parte sea sumisa frente al estilo agresivo de la otra. El gran desafío para el mediador es que ambas logren cambiar el estilo comunicacional para poder alcanzar una comunicación asertiva en el proceso de gestión del conflicto al que se ven enfrentadas.

\section{El razonamiento crítico}

El razonamiento crítico estriba en analizar y evaluar la consistencia de los pensamientos -en especial de aquellas afirmaciones que la sociedad acepta como verdaderas en el contexto de la vida cotidiana- a través del conocimiento y la inteligencia para poder tomar una posición fundada sobre un determinado tema. Uno de los principios del mediador es ser neutral. Esa objetividad necesaria está implícita en esta habilidad, la cual consiste en razonar críticamente en cuanto al seguimiento del proceso y las técnicas a utilizar.

Según Elder y Paul (2005), "es importante resaltar que el desarrollo del pensamiento crítico implica la utilización de estrategias cognitivas, que, sometidas a estándares intelectuales, progresivamente habilitan y aumentan la probabilidad de resultados más oportunos a la hora de pensar" (p. 7). 
Las habilidades que Halpern (1998), según explican Júdex-Orcasitas, Borjas y Torres (2019), identifica como asociadas al razonamiento crítico son:

El razonamiento verbal y escrito, es decir, la capacidad de comprender y utilizar la información que está incluida en nuestro lenguaje cotidiano; el análisis del argumento que permite identificar información relevante, supuestos y creencias; la formulación de hipótesis; la posibilidad de analizar una situación identificando imprecisiones, contradicciones o reconociendo las limitaciones y de la ejecutoria de planes de contingencia, así como la resolución de problemas utilizando múltiples estrategias que pueden implementarse para analizar y resolver un problema de la vida real. (p. 22)

Esta habilidad en la figura del mediador y de las partes se activa cuando ellas se enfrentan a una carencia o necesidad en el momento de gestionar el conflicto. Al detectarse, se despliega esta capacidad como un conjunto de acciones y estrategias que, trabajando en forma conjunta, generan razonamiento crítico sobre determinado hecho, lo que lleva a la toma de decisiones en el proceso de gestión del conflicto (Saiz, 2012). Para Saiz (2012), "el pensamiento crítico es la mayor expresión de un óptimo funcionamiento intelectual" (p. 25). Se produce en el mediador y en las partes una habilidad que les permite vincular elementos argumentativos y explicativos, lo que genera toma de decisiones que, a través del proceso de mediación, no solo los llevan a lograr solucionar un conflicto, sino a transformar la realidad.

\section{El autocuidado}

Esta habilidad es fundamental para que el mediador pueda cumplir su rol de manera eficiente. El proceso de mediación está centrado en la gestión del conflicto que, en principio, siempre tiene una esencia negativa y genera sentimientos adversos en las partes que se enfrentan a él. Por lo tanto, el mediador debe afrontar, en la gran mayoría de los procesos, estos sentimientos por parte de los involucrados y gestionarlos.

El rol del mediador no implica solo el manejo de la gestión que las partes hacen de su conflicto, sino que debe gestionar también sus propias emociones para que no afecten la calidad de su rol. Es habitual que, además, el contexto en el que se realiza el proceso de mediación tenga una connotación negativa, y el mediador debe poder manejarlo de modo que este no influya en su ejercicio profesional.

Uribe (1999) define el autocuidado como

Una práctica que involucra líneas de crecimiento en las que toda persona debe trabajar cotidianamente para tener un desarrollo armónico y equilibrado. Estas líneas de crecimiento que propician un desarrollo integral se relacionan con la dimensión 
emocional, física, estética, intelectual y trascendental del ser, a través del desarrollo de las habilidades afectivas, cognoscitivas y sociales. (p. 16)

El autocuidado tiene ciertos principios que deben ser tenidos en cuenta en el momento de su implementación. En primer lugar, es un acto de vida que permite a la persona, a través de su voluntad, ser sujeto de sus propias acciones. Por otro lado, debe ser una filosofía de vida que acompañe al individuo en su quehacer diario y ser fundamento para el sistema formal e informal en el que interactúa. Y, por último, es una práctica social que implica cierto conocimiento y elaboración del saber, que trae implícitos determinados intercambios y relaciones interindividuales (Uribe, 1999, p. 16).

Debe también —quien pretende cumplirlo—, siguiendo a Uribe (1999), contar con ciertas estrategias para que este autocuidado sea posible:

a) Desarrollar en las personas autoestima, empoderándolas de un modo que les permitan cambiar sus condiciones personales en pro de su cuidado.

b) Involucrar el diálogo de saberes, lo que permite identificar, interpretar y comprender la dinámica del mundo de la vida de las personas.

C) Explorar y comprender la ruptura que existe entre conocimiento, actitudes y prácticas, y tratar de generar propuestas de acción que hagan viable su armonía.

d) Asumir el autocuidado como vivencia cotidiana para poder compartir con las partes la importancia de su cuidado.

e) Contextualizar el autocuidado en la realidad de las partes del proceso, de acuerdo con sus creencias, valores y motivaciones, entre otros elementos.

En este sentido, Campos et al. (2014):

El autocuidado incluye seguir estándares reconocidos para cuidar de uno mismo, (descansar, dormir ...), pero también conviene utilizar un plan de auto-cuidado diseñado individualmente y actualizarlo periódicamente. Es fundamental la auto-monitorización o bien la monitorización a través del apoyo social para conocer el nivel de satisfacción y fatiga de compasión.

Un pobre auto-cuidado puede tener consecuencias negativas como reducir el nivel de satisfacción por compasión, dañar la salud del profesional de ayuda y de sus relaciones, así como dañar a los clientes. (p. 15).

\section{La resiliencia}

La resiliencia puede definirse como "la capacidad de una persona para recobrarse de la adversidad fortalecida y dueña de mayores recursos. Se trata de un proceso activo de resistencia, autocorrección y crecimiento como respuesta a las crisis y desafíos de la vida" (Walsh, 2004, p. 26). Este concepto viene a derribar 
nociones convencionales, tales como que no es posible eliminar los efectos de los traumas tempranos o graves que se han sufrido y que las situaciones adversas siempre tienen, a corto o largo plazo, efectos perjudiciales para el individuo.

Ahora, siguiendo a Walsh (2004), la resiliencia va más allá de sobrevivir al evento adverso, ya que hay personas que quedan atrapadas en este suceso en la condición de víctimas, se retroalimentan en las heridas y se ven impedidas para crecer por el enojo y el sentimiento de culpa. Mientras tanto, quienes poseen la virtud de la resiliencia son capaces de curar sus dolorosas heridas, hacerse cargo de sus vidas y desarrollar una existencia integral y plena (Wolin y Wolin, 1993, p. 8).

El concepto de resiliencia es diferente a los de invulnerabilidad y falta de sufrimiento. La resiliencia implica que la persona logra ganar la batalla contra el elemento adverso, pero en esa batalla está presente el sufrimiento y se trabaja sobre las dificultades a las que se enfrenta como ser vulnerable, tanto en el plano interno como interpersonal (O'Connell, 1994, p. 234).

Tradicionalmente, se ha identificado la condición de superar la adversidad con una concepción individualista que se basa en las virtudes de la persona y que se considera innata. En la actualidad, el concepto de resiliencia se analiza desde una interacción entre lo innato y lo adquirido, con sustento en las relaciones de apoyo como, por ejemplo, la propia familia o recursos externos como maestras, sacerdotes o terapeutas.

O'Connell (1994) comprobó en su investigación que los individuos, cuando soportan padecimientos, emergen de ellos con una energía que, de no haber sido por estos, no hubieran desarrollado. Esto quiere decir que la resiliencia no surge a pesar de la adversidad, sino a causa de esta.

Walsh (2007) realiza un análisis sistémico de la resiliencia con énfasis en la importancia de esta perspectiva. La autora manifiesta que para lograr una verdadera comprensión del término es necesario adoptar una teoría sistémica. Esta teoría amplía la idea de que la adaptación es individual y la inserta en procesos transaccionales más amplios, como la familia y otros sistemas sociales, y presta atención a las influencias mutuas que se producen en tales procesos. Werner (1989, pp. 72-81) manifiesta que en el niño existe una relación estrecha de afecto con un adulto significativo con el que se identifica y recibe de él señales de aliento a la hora de superar sus penurias. Bowlby (1988, p. 119) se aproxima al contexto relacional, pero lo limita siempre a una forma reducida al centrarlo únicamente en una persona significativa del entorno que establece una relación con el menor en riesgo. 
Desde una perspectiva ecológica, la teoría sistémica analiza las distintas esferas de influencia que impactan sobre el riesgo y la resiliencia en el transcurso de la vida de una persona (Rutter, 1987). Desde una perspectiva evolutiva, los mecanismos de superación y adaptación no son considerados riesgos fijos, sino que implican procesos multideterminados que transcurren temporalmente.

La resiliencia es una habilidad básica para gestionar el proceso de mediación en lo que refiere a las partes. Es un proceso comunicativo en el que los protagonistas del conflicto tienen la capacidad de solucionar los problemas cuando actúan de manera constructiva a través de una actitud dinámica y activa. De esta manera, se fortalecen los vínculos entre ellos y con aquellos que, a criterio del mediador y de las partes, puedan ser importantes para lograr una solución en el conflicto; se impulsa la capacidad que tienen como seres humanos para sobreponerse a la adversidad y, a partir de esta adversidad, construir un futuro entre sí durante el proceso. Guisao, Rincón y Arias-Pérez (2017) afirman que es fundamental para el mediador lo que ellos denominan la "capacidad de absorción", esto es, de reconocer "información nueva externa, asimilarla, transformarla y aplicarla con el fin de crear valor". Si bien los autores lo analizan organizacionalmente, esta capacidad se enmarca dentro de la habilidad de resiliencia que debe lograr desarrollar cualquier mediador y desplegarla en las partes para obtener la información de la nueva situación planteada, procesarla y generar una nueva situación aportando valor.

La tarea del mediador en el transcurso de la mediación es comprender en qué puntos se debe potenciar la resiliencia para conseguir resultados más exitosos en la gestión del conflicto y en el aprendizaje necesario que las partes deben hacer de este proceso para poder enfrentar conflictos que se presenten en el futuro.

\section{Conclusiones}

El manejo de las habilidades sociocognitivas tiene un fuerte impacto en el proceso de mediación. Es un tema que debe tener un necesario abordaje interdisciplinario en el que la psicología, la educación, la medicina y el área de gestión de conflictos enriquezcan el análisis desde diferentes perspectivas.

Tanto el mediador como las partes cuentan con algunas de ellas desde el momento de su nacimiento, ya que son innatas, mientras que otras se van desarrollando en función de la realidad de cada individuo. Sin perjuicio de esto, es importante que el mediador tenga en cuenta que dichas habilidades tienen que estar presentes en el proceso y que, de no tenerlas él o las partes, deben ser desarrolladas durante su transcurso. 
Es imprescindible lograr una escucha activa del mediador hacia las partes, de las partes entre sí y con respecto al mediador para poder gestionar cualquier conflicto al que se vean enfrentados.

La empatía es una habilidad indispensable para generar vínculos saludables en la vida cotidiana que se hace también fundamental a la hora de entender a los demás. Asimismo, es imprescindible el autocontrol tanto del mediador como de las partes. Su importancia se acentúa en procesos en los que los conflictos que se gestionan tienen alto nivel emocional. En este sentido, Castellano et al. (2017) afirman:

[La] asertividad permitirá a las personas actuar con base en sus intereses, defenderse sin ansiedad y expresar cómodamente sus sentimientos; ello implica el respeto tanto hacia el propio individuo como hacia los demás, al reconocer también los sentimientos y los derechos de los otros. (p. 58)

El pensamiento creativo es, sin lugar a duda, un método alternativo de resolución de conflictos de la mediación que genera un nuevo camino de entendimiento a nivel social y permite construir otras posibilidades que permitan solucionar los conflictos que se presenten (Baca, 2011).

El razonamiento crítico como habilidad del mediador, tal como afirman Tamayo, Zona y Loaiza $(2015,123)$, es "una actitud frente al conocimiento y hacia la vida" que nos permite enfrentarnos a las situaciones conflictivas y poder resolverlas.

El autocuidado del mediador supone aprender a participar en las dinámicas de la sociedad, en el proceso de mediación y tomar posición frente a las demandas recibidas, sabiendo cuidarse en su rol a través de las estrategias desarrolladas. Además de las prácticas que genere, el mediador debe componer espacios de reflexión con las partes sobre lo que estas saben, viven y sienten en las diferentes situaciones de la vida. De esta manera, pueden ayudar a identificar acciones a favor de su propio autocuidado.

La compasión es una habilidad innata, pero en el desarrollo del individuo, muchas veces debido al contexto, pierde intensidad. Es importante que en el proceso se logre que el mediador y las partes vuelvan a descubrirla, ya que los seres humanos son, por sí mismos, compasivos con el semejante.

Ser resiliente es, en la actualidad, una habilidad que realmente regula la calidad de vida, ya que no se puede evitar enfrentar conflictos, pero sí se puede controlar el modo en que se enfrentan y qué enseñanzas tomar de ellos.

En conclusión, es indudable la importancia que estas habilidades revisten en el proceso de gestión de cualquier conflicto, sin importar su naturaleza. Por 
otro lado, al ser algunas innatas y otras adquiridas, es relevante tener en cuenta que su desarrollo debería hacerse en todos los ámbitos en los que el individuo se desenvuelve, ya sea familiar, educativo, organizacional o cualquier otro.

\section{Agradecimientos}

El presente artículo deriva de la actividad investigativa de quien lo escribe en el terreno de gestión de conflictos, particularmente, en el ámbito de la especialización desde un enfoque interdisciplinario del método. Se profundiza, en esta oportunidad, en las habilidades sociocognitivas como herramientas en el proceso de mediación que permiten a las partes y al propio mediador gestionar de forma constructiva sus conflictos.

\section{Referencias}

Abello, R., Amar, J. y Tirado, D. (2014). Desarrollo infantil y construcción del mundo social. La Imprenta Editores.

Baca, C. (2011). Reseña de los medios a las mediaciones. Comunicación, cultura y hegemonía, J. Martín barbero. Razón y Palabras, 75. http://www.razonypalabra.org.mx/N/N75/monotematiCo_75/07_Baca_M75.pdf

Balletto, S., Briz, M. y Falca, L. (2019). Manual básico de mediación. Universidad Católica del Uruguay.

Barron, F. (1969). Creative person and creative process. Holt, Rinehart \& Winston.

Bowlby, J. (1988). A secure base. Parent - child attachment and healthy human development. Routdlege.

Caballo, V. (2007). Manual de evaluación y entrenamiento de habilidades sociales (7a edición). Siglo XXI Editores.

Camacho E. y Céspedes, N. (2018). Habilidades socio-emocionales: un elemento esencial en el policía como mediador en Colombia. Diversitas: Perspectivas en piscología, 14(2), 279-295. Enlace.

Campos, J., Cardona, J., Bolaños, I. y Cuartero, M. (2014, setiembre). La Fatiga por Compasión en la Práctica de la Mediación Familiar. Hipótesis para una investigación [comunicación]. X Congreso Mundial de Mediación, Génova, Italia. https://www.researchgate.net/publication/279288785_ La_Fatiga_por_Compasion_en_la_Practica_de_la_Mediacion_Familiar_Hipotesis_para_una_investigacion

Caram, M. (2006). Mediación. Diseño de una práctica. Librería Histórica.

Castellano, M., Virviescas, J., Castro, E., Alvarino, C., Pinzón, B. y Gutiérrez, R. (2017). Resolución de conflictos para el fomento de la cultura de la paz: importancia de los medios de comunicación alternos en Colombia. Revista Lasallista de Investigación, 14(1), 56-65. https://doi.org/10.22507/ rli.v14n1a4

Cuesta, O. y Lora, M. (2015). La mediación creativa. Un acercamiento a su construcción conceptual. Revista Luciérnaga, 14(7), 46-63. https://revistas.elpoli.edu.co/index.php/luc/article/view/818

Echeverria, L., Pineda, J., Lafont, T. y Palleres, S. (2020). Impacto de la movilidad internacional en el desarrollo de competencias blandas y su aplicación en el mercado laboral: Un análisis para la 
mejora curricular desde la perspectiva de graduados de ciencias administrativas y contables. Revista Latinoamericana de Estudios Educativos, 3(1), 217-254. https://www.redalyc.org/jatsRepo/270/27063236011/index.html

Elder, L. y Paul, R. (2005). Una guía para los educadores en los estándares de competencia para el pensamiento crítico. Estándares, principios, desempeño indicadores y resultados con una rúbrica maestra en el pensamiento crítico. Fundación para el Pensamiento Crítico.

Fernández-Abascal, E., Jiménez, M. y Martín, M. (2003). Emoción y Motivación. La adaptación humana. Centro de Estudios Ramón Areces.

Font, M., Heras, J., Latorre, J., Nieto, M., Ricarte, J. y Ros, L. (2020). Cognitive avoidance questionnaire: factor structure and psychometric properties. Anales de Psicología / Annals of Psychology, 36(3), 457-467. https://doi.org/10.6018/analesps.397711

Gatti, B. (2005). Habilidades cognitivas y competencias sociales. (M. Rodríguez, trad.). Enunciación, 10(1), 123-132. https://revistas.udistrital.edu.co/index.php/enunc/article/view/462

Gelsi, P. (2011). Transferencia y empatía. Ciencias Psicológicas, 5(1), 117-122. https://revistas.ucu.edu. uy/index.php/cienciaspsicologicas/article/view/103

Goleman, D. (1995). Inteligencia emocional. Kairós.

Guisao, S., Rincón, L., y Arias-Pérez, J. (2018). Capacidad de tecnologías de información y desempeño organizacional: efecto mediador de la capacidad de absorción. Cuadernos de Administración, 30(55). https://doi.org/10.11144/Javeriana.cao.30-55.ctido

Hirsch, A., Izarra, D. y Navia, C. (2020). Rasgos Propuestos por Académicos de Posgradocon Respecto a lo que Significa Ser un Buen Profesor Universitario. Trayectorias, (51), 3-24. http://www.trayectorias.uanl.mx/51/index.htm

Iese Insight (2014). Negociación: el poder de la escucha activa. Las habilidades necesarias para cerrar un buen trato. Iese Insight (20), pp. 67-71.

Izquierdo, G. (2010). Ensayo sobre el proceso de la comunicación efectiva. Atlantic International University. https://bit.ly/3uP4Ont

Júdex-Orcasitas, J., Borjas, M. y Torres, E. (2019). Evaluación de las habilidades del pensamiento crítico con la mediación de las TIC, en contextos de educación media. Reidocrea, 8, 21-34. https:// www.ugr.es/ reidocrea/8-4.pdf

Latorre, M. (2020). iEsto también pasará! (Mensaje a los millenials, centenials y a los más mayores). Educa UMCH, 1(15), 5-22. https://doi.org/10.35756/educaumch.202015.133

Martí, F. (2020). Cómo mejorar nuestra comunicación con los demás en el trabajo. Gestión Práctica de Riesgos Laborales, (181), 52-54.

Melendro, M. y Montserrat, C. (2017). ¿Qué habilidades y competencias se valoran de los profesionales que trabajan con adolescencia en riesgo de exclusión social? Análisis desde la acción socioeducativa. Educación XXI, 20(2), 113-135. http://revistas.uned.es/index.php/educacionXX1/article/ view/19034

O'Connell, G. (1994). Resilient adults: overcoming a cruel past. Jossey-Bass. 
Ortiz, L. y Gaeta, M. (2019). Desarrollo socio-afectivo en la educación media superior: el papel del contexto académico. Revista Panamericana de pedagogía, saberes y quehaceres del pedagogo, (27), 149-167.

Pesqueira, J. y Ortiz, A. (2018). Mediación asociativa y cambio social. El arte de lo posible. Universidad de Sonora.

Rancer, A. y Avtgis, T. (2006). Argumentative and Aggressive Communication: Theory, Research and Application. Sage.

Rutter, M. (1987). Psychosocial resiliencie and protective mechanisms. American Journal of Orthopsychiatry, 57(3), 316-331.

Saiz, C. (2012). Pensamiento crítico: conceptos básicos y actividades. Ediciones Pirámide.

Sellers, M. (2019) Derecho, razón y emoción. (G. Arias, trad.). Revista Filosofía, 18(2), 235-260. https:// doi.org/10.18273/revfil.v18n2-2019012

Slabbert, J. (1994). Creativity in education revisited: reflection in aid of progression. The Journal of Creative Behavior, 28(1), 60-69.

Sternberg, R. y O'Hara, L. (2005). Creatividad e inteligencia. CIC. Cuadernos de Comunicación e Información, (10), 113-149. https://revistas.ucm.es/index.php/CIYC/article/view/CIYC0505110113A

Tamayo, O. Zona, R., Loaiza, Y. (2015). El pensamiento crítico en la educación. Algunas categorías centrales en su estudio. Revista Latinoamericana de Estudios Educativos, 11(2), 111-133.

Uribe, T. (1999). El autocuidado y su papel en la promoción de la salud. Investigación y Educación en Enfermería, 17(2), 109-118. https://revistas.udea.edu.co/index.php/iee/article/view/16870

Walsh, F. (2004). Resiliencia familiar: estrategias para su fortalecimiento. Amorrortu.

Walsh, F. (2007). Traumatic loss and major disasters: strengthening family and community resilience. Family Process, 46(2), 207-227.

Walters, R. (28/10/2020). Las 4 aptitudes imprescindibles del líder del futuro. Especial directivos. https://bit.ly/38PRttJ

Werner, E. (1989). High-risk children in young adulthood: a longitudinal study from birth to 32 years. American Journal of Orthopsychiatry, 59(1), 72-81.

Wolin, S. y Wolin, S. (1993). The resilient self: how survivors of troubled families rise above adversity. Villard Books. 